\title{
Size Effect in Plasmon Resonance of Metallic Nanoparticles: RPA versus COMSOL
}

\author{
K. KLUCZYK AND W.A. JACAK* \\ Department of Quantum Technologies, Faculty of Fundamental Problems of Technology, \\ Wrocław University of Technology, Wybrzeże Wyspiańskiego 27, 50-370 Wrocław, Poland
}

\begin{abstract}
Size effect for plasmon resonance in metallic nanoparticles has been studied by finite element method solution of the Maxwell equations (COMSOL), by the Mie approach and microscopic random phase approximation model. Comparison with $\mathrm{Au}, \mathrm{Ag}$ nanoparticles experimental data for light extinction in colloidal solutions with different particle sizes is presented for the three types of approach.
\end{abstract}

DOI: 10.12693/APhysPolA.129.A-83

PACS: 1. 36.40.Gk, 73.20.Mf

\section{Introduction}

Optical properties of small metallic particles even with complicated shape can be studied using numerical methods [1]. In the present report we compare the results obtained by the finite element method utilizing COMSOL system with the microscopic random phase approximation (RPA) modeling of surface plasmons and with the Mie approach. Results are discussed with comparison to experimental data for gold and silver nanoparticles.

\section{RPA modeling of plasmons in metallic particles}

Upon the dipole approximation the optical response of metallic nanosphere resolves itself to only dipole type surface plasmon oscillations, which can be described by RPA equation [2] of oscillatory form with damping caused by electron scattering on other electrons, phonons, admixtures and on metallic nanoparticles (MNP) boundary

$$
\frac{1}{\tau_{0}}=\frac{v_{F}}{2 \lambda_{b}}+\frac{C v_{F}}{2 a},
$$

where $\lambda_{b}$ is the electron mean free path in bulk metal (including scattering of electrons on other electrons, impurities and phonons [3]) and $C$ is the constant of unity order. Electron density oscillations are the source of the electromagnetic radiation, and thus the related energy losses also contribute to plasmon attenuation. This effect can be expressed by the Lorentz friction effective field

$$
\boldsymbol{E}_{L}=\frac{2}{3 c^{3}} \frac{\partial^{3} \boldsymbol{D}(t)}{\partial t^{3}}
$$

$\boldsymbol{D}(t)$ - plasmon dipole. Upon the perturbation approximation one can define the renormalized damping term

\footnotetext{
* corresponding author; e-mail: witold.aleksander. jacak@pwr.edu.pl
}

including Lorentz friction [4]:

$$
\frac{1}{\tau}=\frac{1}{\tau_{0}}+\frac{\omega_{1}}{3 \sqrt{\varepsilon}}\left(\frac{\omega_{p} a}{c \sqrt{3}}\right)^{3} .
$$

The contribution to attenuation of plasmons caused by the irradiation losses (upon the perturbation approach) scales as $a^{3}$, and therefore, for large nanospheres, the radiative losses dominate plasmon damping. On the other hand, for small nanospheres scattering type damping, which scales as $\frac{1}{a}$, is more important. One can observe thus the crossover is size dependence of damping at $c a .12 \mathrm{~nm}$ for $\mathrm{Au}$ [4], as illustrated in Fig. 1.

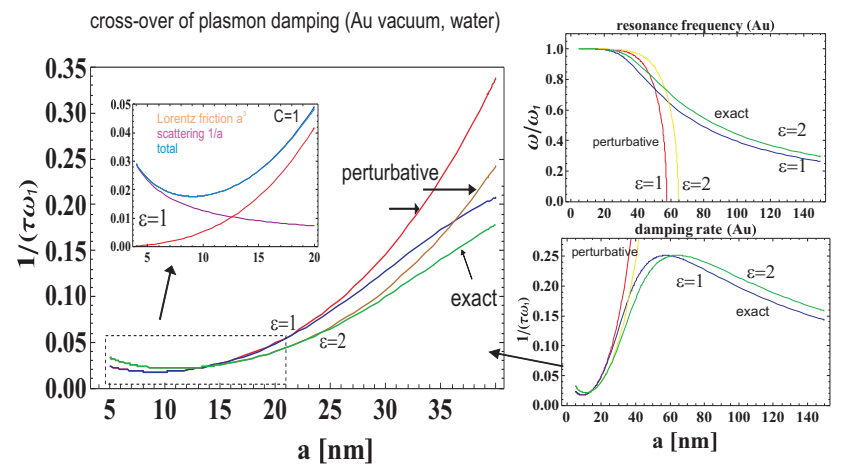

Fig. 1. Crossover in size dependence of surface plasmon damping in MNPs (Au nanoparticles in vacuum and in water); right parts present the exact selffrequency and damping rate according to Eq. (1).

The perturbation approach resulting in $a^{3}$ growth of plasmon damping is, however, not justified for radii $a>30 \mathrm{~nm}$ (for $\mathrm{Au}$ in vacuum) and the exact form of the Lorentz friction must be accounted for in large MNP limit, as described in [5]. Exact solution of the dynamic equation with the Lorentz friction exhibit saturation of the damping growth instead of the $\sim a^{3}$ increase, as depicted in Fig. 1 (right). The connection between the damping rate and the resonance frequency shift in perturbative approximation is described by the damped 
oscillator-type formula

$$
\omega_{1}^{\prime}=\sqrt{\omega_{1}^{2}-\frac{1}{\tau^{2}}}
$$

where $\omega_{1}^{\prime}$ is the shifted resonance frequency. Nevertheless, the perturbation approach is valid only for particle radii lower than $30 \mathrm{~nm}(\mathrm{Au})$, and for larger radii neither damping nor self-frequency is given by the above formulae and the exact solutions differ significantly from the approximated perturbation values. The analytical form of the exact solution for the damping and self-frequency including Lorentz friction is as follows [5] (the exponents of solution $\sim \mathrm{e}^{\mathrm{i} \Omega t}$ for self-modes):

$$
\begin{aligned}
\Omega_{1} & =-\frac{\mathrm{i}}{3 l}-\frac{\mathrm{i} 2^{1 / 3}(1+6 l q)}{3 l \mathcal{A}}-\frac{\mathrm{i} \mathcal{A}}{2^{1 / 3} 3 l}=\mathrm{i} \alpha \in \mathrm{Im}, \\
\Omega_{2} & =-\frac{\mathrm{i}}{3 l}+\frac{\mathrm{i}(1+\mathrm{i} \sqrt{3})(1+6 l q)}{2^{2 / 3} 3 l \mathcal{A}}+\frac{\mathrm{i}(1-\mathrm{i} \sqrt{3}) \mathcal{A}}{2^{1 / 3} 6 l}= \\
\omega & +\mathrm{i} \frac{1}{\tau}, \\
\Omega_{3} & =-\omega+\mathrm{i} \frac{1}{\tau}=-\Omega_{2}^{*},
\end{aligned}
$$

where $\mathcal{A}=\sqrt[3]{\mathcal{B}+\sqrt{4(-1-6 l q)^{3}+\mathcal{B}^{2}}}, q=1 /\left(\tau_{0} \omega_{1}\right)$, $\mathcal{B}=2+27 l^{2}+18 l q$ and $l=\frac{2}{3 \sqrt{\varepsilon}}\left(\frac{a \omega_{p}}{c \sqrt{3}}\right)^{3}$. The functions $\omega$ and $\frac{1}{\tau}$ (in dimensionless units, i.e., divided by $\omega_{1}$ ) are plotted in Fig. 1 (right) versus nanosphere radius $a$, for illustration. Exact inclusion of the Lorentz friction indicates that the radiative losses and the self-frequencies are complicated function of particle radius as given by Eq. (1) (by imaginary and real parts of $\Omega$, correspondingly) and damping can be approximated by simple $\sim a^{3}$ growth of attenuation only in relatively narrow size window, e.g., ca. $12-30 \mathrm{~nm}$ for $\mathrm{Au}$ in vacuum $[4,5]$.

\section{Numerical modeling of plasmon resonances in MNP}

The weak side of numerical solution of the Maxwell equations consists in phenomenological assumption of the dielectric function for MNP similarly as within the Mie approach. The advantage of the COMSOL calculation is its flexibility versus geometry and material setup. COMSOL (similarly as the Mie calculus) includes all multipole contribution important for larger MNP beyond the dipole approximation. For particles of size larger than $\sim 0.2$ of incident light wavelength, the quadrupole mode contributes. Figure 2 shows the comparison of optical characteristics of three $\mathrm{Au}$ nanospheres with radii 20, 70, and $140 \mathrm{~nm}$ and extinction spectra for $\mathrm{Au}, \mathrm{Ag}$ and $\mathrm{Cu}$ nanospheres found by COMSOL. The influence of nanosphere radii on the optical response is seen from the extinction spectra where the contribution of multipoles is noticeable.

The numerical COMSOL resonances were compared with experimental resonances taken from extinction spectra measured for colloidal solutions of metallic nanospheres with various dimensions. The resonances

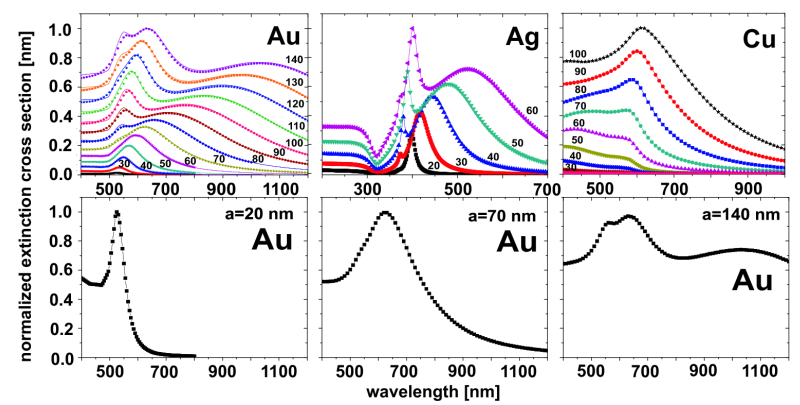

Fig. 2. Extinction spectra calculated for $\mathrm{Au}, \mathrm{Ag}$ and $\mathrm{Cu}$ nanospheres surrounded by water by COMSOL (points) in comparison to spectra obtained by the Mie theory (solid lines) for various size of nanospheres of these metals, $\mathrm{Au}(30-140 \mathrm{~nm}), \mathrm{Ag}(20-60 \mathrm{~nm}), \mathrm{Cu}$ (20-100 nm) (upper). Extinction spectra for three $\mathrm{Au}$ nanospheres with radii 20,70 , and $140 \mathrm{~nm}$ with features indicating multipole contribution (lower).

have been compared also with theoretical predictions within RPA approach including exact calculation and perturbative approximation for the Lorentz friction. Comparison of results for Au (presented in Fig. 3) shows good agreement of exact RPA calculations with experiment and numerical estimations. The perturbative approximation for the Lorentz friction (resulting in $\sim a^{3}$ damping growth) is valid only for spheres smaller than ca. $30 \mathrm{~nm}$ (for $\mathrm{Au}$, vacuum), for larger spheres the predicted increase of attenuation upon perturbation approach is much faster than in experiment and numerical calculations and produces an artifact - the overdamped regime when an oscillating solution disappears. Let us emphasize, however, that the exact inclusion of the Lorentz friction, as given by Eq. (1), is free of this artifact. It means that the oscillating solution in larger MNP is not of damped harmonic oscillator form (as that for perturbative approach), but corresponds to the distinct dynamic equation of the third order differential equation caused by the Lorentz friction.

In the case of $\mathrm{Ag}$ nanoparticles the experimental data are shifted to longer wavelengths in comparison to both our numerical and theoretical predictions. Probably it is the consequence of silver nanoparticle synthesis method, which could leave on the silver surface residual amount of organic surfactant (citrate) or other chemically and optically active molecules. This effect may be verified in COMSOL by introducing of specially defined dielectric coating to the model MNP. Figure 3 shows changes of the plasmon resonance wavelength due to varying thickness of dielectric shell with refractive index $n=1.5$ and due to varying shell refractive index with shell thickness $10 \mathrm{~nm}$. Both increasing shell thickness and increasing refractive index of the shell material cause redshift of resonance wavelength. The best fit between theory and experimental data is obtained using refractive index 1.5 and thickness of the model coating layer of $10 \mathrm{~nm}$. 

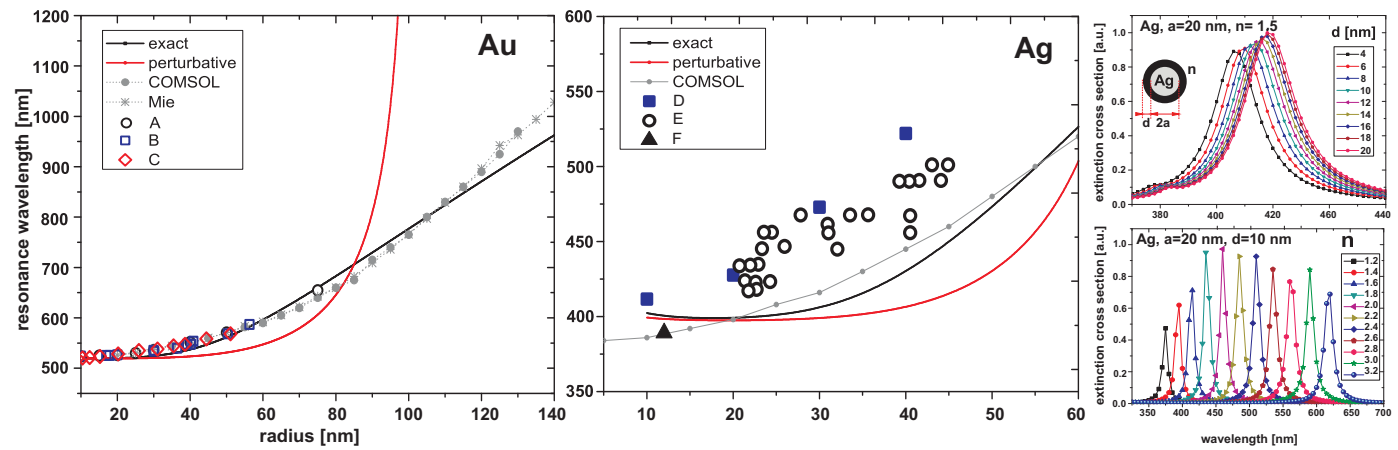

Fig. 3. Plasmon self-frequencies for plasmon in MNPs ( $\mathrm{Au}$ and $\mathrm{Ag})$ found by RPA, COMSOL Mie and from experiment - A and D, B - after [6], C - after [7], E - after [8], F - after [9]. Perfect agreement Mie-COMSOL corresponds to the same dielectric function assumed as the prerequisite for both fully equivalent solutions of the Maxwell equations. Discrepancy for Ag may be related with silver MNP surface chemical modification, which can be modeled by additional outer layer (right parts).

\section{Comparison with the size effect in the Mie theory}

To explain the crossover in the size dependence of the experimentally observed red-shift of plasmon resonance in MNPs with growing nanosphere radius, within the Mie theory two regimes are usually considered [10]: the intrinsic size effect (for $a<20 \mathrm{~nm}$, for $\mathrm{Au}$ ) and the extrinsic one (for $a>20 \mathrm{~nm}$ ). The intrinsic size effect is referred to the dipole approximation of the Mie response which is justified for nanosphere radius well smaller than the plasmon resonance wavelength. For relatively small particles, as in the case of the intrinsic size effect, the model dielectric function includes electron scattering which gives $\sim \frac{1}{a}$ red-shift of the dipole Mie resonance. For larger nanospheres (approximately for $a>20 \mathrm{~nm}$, for $\mathrm{Au}$ ) when in experiment there is observed the resonance redshift rising with $a$ growth, the extrinsic mechanism is suggested upon the Mie theory [10-12]. The extrinsic regime resolves itself in the Mie theory to the inclusion of the multipole mixing in e-m response. To obtain a coincidence with the experimentally observed size effect in the red-shift of plasmon resonance in larger nanospheres the irradiation corrections to the dielectric function have been introduced proportional to the number of electrons, thus $\sim a^{3}[13]$. This overestimates, however, the radiative damping. For small MNP radii the dipole approximation holds - thus inclusion of electron scattering gives reasonable agreement with experimental observations. Nevertheless, for larger radii (approximately for radii $a>20 \mathrm{~nm}$, for $\mathrm{Au}$ ) the observed experimentally resonance red-shift is not proportional to $\frac{1}{a}$, though the dipole limit still holds. The Lorentz friction induced irradiation losses strongly contribute to plasmon energy dissipation for $a>12 \mathrm{~nm}$ (for $\mathrm{Au}$ in vacuum). For such radii the irradiation losses dominate plasmon damping with a factor higher than 1 ( $c a .5$ at $a=20 \mathrm{~nm})$. This was not included in the conventional Mie theory upon the intrinsic size effect.

The irregular size effect of plasmon damping caused by the Lorentz friction, if accounted for accurately $(c f$. for-

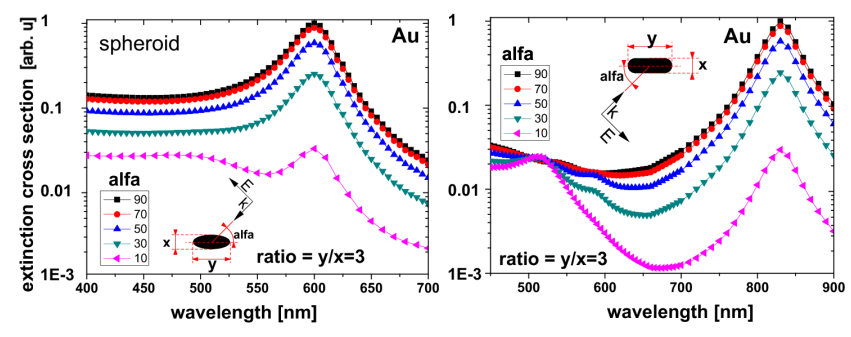

Fig. 4. Extinction spectra for $\mathrm{Au}$ spheroid and nanorod for varying polarization of incident light (COMSOL). Longintudinal (electric field parallel to long axis) and transversal (electric field perpendicular to long axis) plasmon modes are visible.

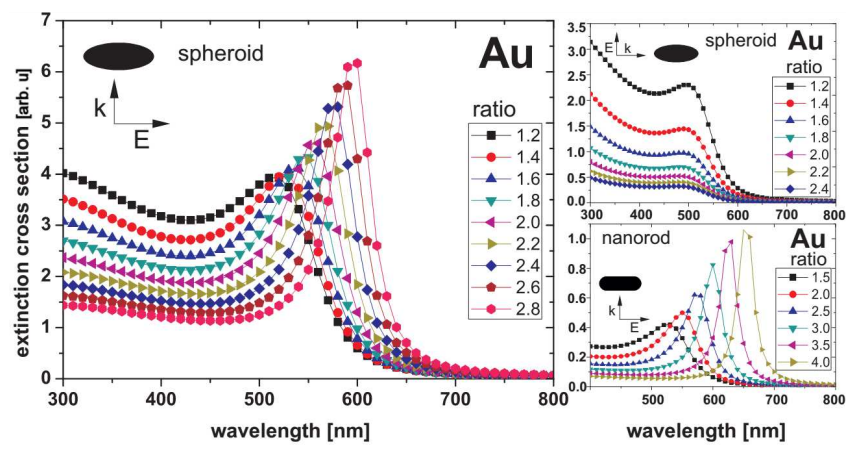

Fig. 5. Extinction spectra calculated for Au spheroid and nanorod with varying aspect ratio (COMSOL).

mula (1)), well explains the experimentally observed also irregular (i.e., not proportional to $a^{3}$ ) size effect for the red-shift. This Lorentz friction induced correction mixes, however, with the extrinsic size effect due to the multipole contributions, but rather for radii $a>60 \mathrm{~nm}(\mathrm{Au})$ significantly exceeding the previously suggested limiting $20 \mathrm{~nm}[10,11,13]$. The quadrupole contribution (and the higher multipoles at larger radii) results in the deformation and larger broadening of the extinction fea- 
tures not allowing its Lorentzian form any longer (the higher energy quadrupole assistant broad peak occurs first at smaller wavelength in association to the dipole peak broadened and red-shifted by the Lorentz friction). In the experiment it is visible for $\mathrm{Au}$ (at $75 \mathrm{~nm}$ ) and $\mathrm{Ag}$ (at 30-40 nm) [14, 15]. The multipole corrections cannot be avoided for radii $a>60 \mathrm{~nm}(\mathrm{Au})$, as is evident by deformation of the Lorentzian shape describing extinction features accompanied by their significant broadening, for Au observed at $a=75 \mathrm{~nm}[14,15]$.

\section{Summary}

The accurate microscopic analysis of the Lorentz friction carried out within the RPA approach supports modeling of the dielectric function needed in the Mie theory (and in COMSOL) and thus can well be utilized to prolong the intrinsic size-effect upon the Mie approach beyond the $20 \mathrm{~nm}$ size limit to larger radii for which the dipole approximation still holds (up to approximately $60 \mathrm{~nm}$ for $\mathrm{Au}$ ). One should note however, that since dielectric function simultaneously represents all energy dissipation channels in a combined manner (assumed phenomenologically damping is 10 times greater than $\frac{v_{F}}{2 \lambda_{b}}$ ), details of the size effect caused by the exact form of the Lorentz friction (out of the scope of the Mie theory as of yet [13]) are required to be included to the modeled dielectric function. Thus, the specific size dependence of the damping time rate for plasmons due to the Lorentz friction for electron density dynamics in nanospheres may be employed to explain the observed size-effect of plasmon resonance in the radius window 10-60 $\mathrm{nm}$ (for $\mathrm{Au}$ ).

\section{Acknowledgments}

Support from NCN project no. 2011/03/D/ST3/02643 and NCBiR project no. PL-TW/1/2013/23 is acknowledged.

\section{References}

[1] A. Taflove, S.C. Hagness, Computational Electrodynamics, Artech House, 3. Boston 2000.

[2] J. Jacak, J. Krasnyj, W. Jacak, R. Gonczarek, A. Chepok, L. Jacak, Phys. Rev. B 82, 035418 (2010).

[3] M.L. Brongersma, J.W. Hartman, H.A. Atwater, Phys. Rev. B 62, R16356 (2000).

[4] W.A. Jacak, J. Phys. Chem. C 119, 6749 (2015).

[5] W.A. Jacak, Opt. Express 23, 4472 (2015).

[6] W. Haiss, Nguyen TK Thanh, J. Aveyard, D.G. Fernig, Anal. Chem. 79, 4215 (2007).

[7] P.N. Njoki, I.-Im S. Lim, D. Mott, Hye-Young Park, Bilal Khan, Suprav Mishra, Ravishanker Sujakumar, Jin Luo, Chuan-Jian Zhong, J. Phys. Chem. C 111, 14664 (2007).

[8] J.J. Mock, M. Barbic, D.R. Smith, D.A. Schultz, S. Schultz, J. Chem. Phys. 116, 6755 (2002).

[9] Ai Leen Koh, Kui Bao, Imran Khan, W.E. Smith, G. Kothleitner, P. Nordlander, S.A. Maier, D.W. McComb, ACS Nano 3, 3015 (2009).

[10] S. Link, M.A. El-Sayed, Int. Rev. Phys. Chem. 19, 409 (2000).

[11] S. Link, M.A. El-Sayed, J. Phys. Chem. B 103, 4212 (1999).

[12] K. Kolwas, A. Derkachova, M. Shopa, J. Quantitat. Spectrosc. Radiat. Transfer 110, 1490 (2009).

[13] K. Kolwas, A. Derkachova, J. Quantitat. Spectrosc. Radiat. Transfer 114, 45 (2013).

[14] W. Jacak, J. Krasnyj, J. Jacak, R. Gonczarek, A. Chepok, L. Jacak, D.Z. Hu, D. Schaadt, J. Appl. Phys. 107, 124317 (2010).

[15] C. Sönnichsen, T. Franzl, T. Wilk, G. von Plessen, J. Feldmann, New J. Phys. 4, 93 (2002). 